\title{
Computation of a Multivariate $F$ Distribution*
}

\author{
By D. E. Amos and W. G. Bulgren
}

\begin{abstract}
Methods for evaluating the joint cumulative probability integral associated with random variables $F_{k}=\left(X_{k} / r_{k}\right) /(Y / s), k=1,2, \cdots, n$, are considered where the $X_{k}$ and $Y$ are independently $\chi^{2}\left(r_{k}\right)$ and $\chi^{2}(s)$, respectively. For $n=2$, series representations in terms of incomplete beta distributions are given, while a quadrature with efficient procedures for the integrand is presented for $n \geqq 2$. The results for $n=2$ are applied to the evaluation of the correlated bivariate $F$ distribution.
\end{abstract}

Introduction. If $X_{k}, k=1, \cdots, n$, and $Y$ are independent $\chi^{2}$ random variables with $r_{k}, k=1, \cdots, n$, and $s$ degrees of freedom, respectively, then the random variables

$$
F_{k}=\frac{X_{k} / r_{k}}{Y / s}, \quad k=1, \cdots, n,
$$

have a joint cumulative distribution $P\left(0 \leqq F_{1} \leqq f_{1}, \cdots, 0 \leqq F_{n} \leqq f_{n} \mid s, r_{k}, k=\right.$ $1, \cdots, n$ ) given by

$$
I_{n}(\hat{\alpha}, \hat{c}, \beta)=\int_{0}^{\infty} \frac{e^{-z} z^{\beta-1}}{\Gamma(\beta)} \prod_{k=1}^{n} \frac{\gamma\left(\alpha_{k}, c_{k} z\right)}{\Gamma\left(\alpha_{k}\right)} d z, \quad \beta>0, \alpha_{k}>0,
$$

where

$$
c_{k}=f_{k} \alpha_{k} / \beta, \quad k=1, \cdots, n, \quad \alpha_{k}=r_{k} / 2 \text { and } \beta=s / 2
$$

and the incomplete gamma function is defined by

$$
\gamma(\alpha, x)=\int_{0}^{x} e^{-t} t^{\alpha-1} d t, \quad \alpha>0, x \geqq 0 .
$$

This distribution arises in many applications, most of which are special cases of (1). Among these are the inverted Dirichlet distribution, the maximum $F$ distribution or multivariate $t^{2}$ distribution [25, p. 159] and an important class where $\alpha_{k}=\alpha$ for all $k$. References [5], [6], [7], [10], [15], [16], [18], [20] contain recent results which utilize this multivariate $F$.

The analysis below forms the basis for a procedure by which $I_{n}$ can be evaluated numerically over a wide range of parameters.

Analysis. We first take the case where $\mu \equiv \beta+\sum_{k=1}^{n} \alpha_{k}>1$ and show that the integrand

Received June 25, 1970, revised April 23, 1971.

AMS 1969 subject classifications. Primary 6525; Secondary 6231.

Key words and phrases. Dirichlet distribution, maximum $F$ distribution, multivariate $t^{2}$ distribution, $F$ with correlation, ranking and selection.

* This work was supported in part by the United States Atomic Energy Commission.

Copyright $\odot$ 1972, American Mathematical Society 


$$
g(z)=\frac{e^{-z} z^{\beta-1}}{\Gamma(\beta)} \prod_{k=1}^{n} \frac{\gamma\left(\alpha_{k}, c_{k} z\right)}{\Gamma\left(\alpha_{k}\right)}
$$

is bell-shaped with a single maximum at $z_{0}>0$. The integration then proceeds from $z_{0}$ to the left and right in the form.

$$
I_{n}=R_{1}+\sum_{i=0}^{N_{1}} \int_{s_{0}-(i+1) \sigma}^{z_{0}-i \sigma} g(z) d z+\sum_{i=0}^{N_{2}} \int_{s_{0}+i \sigma}^{z_{0}+(i+1) \sigma} g(z) d z+R_{2},
$$

where $z_{i}=z_{0}-(i+1) \sigma$ and $R_{1}$ are replaced by zero if $z_{i}$ is negative. This formula sums quadratures over lengths $\sigma$ which approximate the "spread" of $g(z)$. This step is necessary in order to include a large, but not excessive, portion of the area when parameters vary widely. $R_{1}$ and $R_{2}$ are truncation errors given by

$$
R_{1}=\int_{0}^{z_{L}} g(z) d z, \quad R_{2}=\int_{z \sigma}^{\infty} g(z) d z
$$

which can be bounded in the form

$$
\begin{aligned}
R_{1}<B_{1} & =0 & & \text { if } z_{L}=0, \\
& =\frac{\gamma\left(\beta, z_{L}\right)}{\Gamma(\beta)} \prod_{k=1}^{n} \frac{\gamma\left(\alpha_{k}, c_{k} z_{L}\right)}{\Gamma\left(\alpha_{k}\right)} & \text { if } z_{L}>0, & R_{2}<B_{2}=\frac{\Gamma\left(\beta, z_{U}\right)}{\Gamma(\beta)},
\end{aligned}
$$

where $z_{L}=\max \left\{0, z_{0}-\left(N_{1}+1\right) \sigma\right\}$ and $z_{U}=z_{0}+\left(N_{2}+1\right) \sigma$. With these bounds, a relative error test (which can be made after each quadrature addition) is appropriate for the truncation error since

$$
\frac{R_{1} \text { or } R_{2}}{\text { exact sum }} \leqq \frac{R_{1} \text { or } R_{2}}{\text { accumulated sum }}<\frac{B_{1} \text { or } B_{2}}{\text { accumulated sum }} \text {. }
$$

The length $\sigma$ over which each quadrature is taken is estimated by the Laplace method for an asymptotic form. That is, we write $y(z)=\ln g(z), y^{\prime}\left(z_{0}\right)=0$,

(5) $I_{n}=\int_{0}^{\infty} e^{y(z)} d z \sim e^{y\left(z_{0}\right)} \int_{-\infty}^{+\infty} \exp \left\{-\frac{1}{2}\left|y^{\prime \prime}\left(z_{0}\right)\right|\left(z-z_{0}\right)^{2}\right\} d z=e^{y\left(z_{0}\right)}\left(\frac{2 \pi}{\left|y^{\prime \prime}\left(z_{0}\right)\right|}\right)^{1 / 2}$

and

$$
\sigma=\left(\left|y^{\prime \prime}\left(z_{0}\right)\right|\right)^{-1 / 2}
$$

Now, we show that there is only one maxima of $g(z)$, give an equation for $z_{0}$ and compute $y^{\prime \prime}\left(z_{0}\right)$ for (6). Logarithmic differentiation of (2) together with the confluent series form of $\gamma(\alpha, x)$,

(7) $\gamma(\alpha, x)=\frac{e^{-x} x^{\alpha}}{\alpha} \Phi(1,1+\alpha ; x), \quad \Phi(a, c ; x)=\sum_{k=0}^{\infty} \frac{(a)_{k} x^{k}}{(c)_{k} k !}, \quad c \neq 0,-1, \cdots$, yield

$$
y^{\prime}(z)=\frac{g^{\prime}(z)}{g(z)}=\left[-1+\frac{\beta-1}{z}+\sum_{k=1}^{n} \frac{\alpha_{k}}{z \Phi\left(1,1+\alpha_{k} ; c_{k} z\right)}\right] .
$$

Since $\mu>1$ guarantees that $g(0)=0$, the nonzero extrema occur at the roots of

$$
z=\beta-1+\sum_{k=1}^{n} \frac{\alpha_{k}}{\Phi\left(1,1+\alpha_{k} ; c_{k} z\right)} \equiv f(z) \text {. }
$$


The relations

$$
\begin{aligned}
\Phi^{\prime}(1,1+\alpha ; x) & =\frac{1}{1+\alpha} \Phi(2,2+\alpha ; x)>0, \quad x \geqq 0, \alpha>0, \\
\Phi(1,1+\alpha ; x) & \sim \Gamma(1+\alpha) e^{x} x^{-\alpha}, \text { for } x \rightarrow \infty,
\end{aligned}
$$

establish the monotone decreasing behavior of the right side of (8) and hence the uniqueness of the root $z_{0}>\max \{0, \beta-1\}$ for $\mu>1$.

Since

$$
g(0)=0, \quad g(z)>0 \quad \text { for } z>0, \quad \text { and } \quad \lim _{z \rightarrow \infty} g(z)=0,
$$

$g\left(z_{0}\right)$ is a maximum of $g(z)$ and

$$
y^{\prime \prime}\left(z_{0}\right)=-\frac{1}{z_{0}}-\sum_{k=1}^{n} \frac{\alpha_{k} c_{k}}{\left(1+\alpha_{k}\right) z_{0}} \frac{\Phi\left(2,2+\alpha_{k} ; c_{k} z_{0}\right)}{\Phi^{2}\left(1,1+\alpha_{k} ; c_{k} z_{0}\right)} \text {. }
$$

If $1>\mu>0$, the integral $I_{n}$ exists but (8) has no solution and $g^{\prime}<0$. The integrand is therefore monotone decreasing from $\infty$ to 0 and the integration scheme must account for the infinite singularity at $z=0$ when $z=0$ is in the interval of integration. If $2>\mu>1, g(0)=0$ but the infinite derivative of $g(z)$ at $z=0$ causes polynomial type integration schemes to converge very slowly. For $\mu \geqq 2$ this problem is less severe. In many cases, the truncation on the left completely eliminates the problem of singular behavior. If $\mu=1$, no singularity at $z=0$ is encountered, and the integrand decreases monotonically to zero.

Computational Considerations. Due to the wide range of numbers which can be generated, the integrand $g(z)$ is conveniently evaluated in the form

$$
g(z)=e^{n(s)} \prod_{k=1}^{n} \frac{\gamma\left(\alpha_{k}, c_{k} z\right)}{\Gamma\left(\alpha_{k}\right)}
$$

to prevent underflow or overflow during a direct evaluation. Here

$$
h(z)=-z+(\beta-1) \ln z-\ln \Gamma(\beta)
$$

and the ratios $\gamma / \Gamma$ are generated by the following scheme. If $x<1+\alpha$, we use.(7) for $\Phi$ in the form

$$
\begin{gathered}
\Phi(1, c ; x)=\sum_{k=1}^{N} A_{k}+R_{N}, \quad R_{N} \leqq \frac{A_{N+1}}{1-x /(c+N)}, \quad x<c, \\
A_{1}=1, \quad A_{k+1}=A_{k} x /(c+k-1), \quad k=1,2, \cdots, N,
\end{gathered}
$$

with $c=1+\alpha$ and compute $\gamma(\alpha, x) / \Gamma(\alpha)$ from (7),

$$
\frac{\gamma(\alpha, x)}{\Gamma(\alpha)}=\Phi(1,1+\alpha ; x) \exp \{-x+\alpha \ln x-\ln \Gamma(\alpha+1)\}
$$

On the other hand, if $x \geqq 1+\alpha$, we compute $K$ so that $c=1+\alpha+K>x$, generate $\Phi$ as above and recur backward with

$$
\begin{aligned}
y_{K} & =S \Phi(1,1+\alpha+K ; x), \\
y_{k-1} & =\frac{x}{\alpha+k} y_{k}+S, \quad k=K, K-1, \cdots, 1,
\end{aligned}
$$


which is a modification of the two term recurrence relation for $\gamma$. Then, $y_{0}=S \Phi(1,1+\alpha ; x)$ where $S$ is a scale factor on the order of the underflow limit of the machine. Finally,

$$
\frac{\gamma(\alpha, x)}{\Gamma(\alpha)}=(\exp \{-x+\alpha \ln x-\ln \Gamma(\alpha+1)\} / S) \cdot y_{0}
$$

takes care of the scaling. Refinements to take care of the tails of $\gamma / \Gamma$ make the routine faster and eliminate unnecessary computation when the spread of $g(z)$ is large and some ratios in (11) are 1 to the word length of the machine. Thus, we seek $X(\alpha)$ such that, for $x>X, \gamma(\alpha, x) / \Gamma(\alpha)=1$ to the word length of the machine. This value can be estimated from the asymptotic expansion

$$
\frac{\gamma(\alpha, x)}{\Gamma(\alpha)} \sim 1-\frac{\alpha e^{-x} x^{\alpha-1}}{\Gamma(\alpha+1)}=1-10^{-E},
$$

where we take $x=c \alpha$ and solve for $c$ in the relation

$$
F(c)=-\alpha(c-1)+(\alpha-1) \ln c+E \ln 10+\frac{1}{2} \ln \alpha=0
$$

after using the Stirling approximation for $\Gamma(\alpha+1)$. If we take $c=1$ to start Newton's method, the convergence is from above and the termination at $c_{0}$ always provides a conservative estimate of $X(\alpha)=c_{0} \alpha$.

Values of $X(\alpha)$ were generated and an empirical form

$$
X(\alpha)=\frac{c_{1} \alpha^{3}+c_{2} \alpha^{2}+c_{3} \alpha+c_{4}}{\alpha^{2}+c_{5} \alpha+c_{\beta}}
$$

was fitted to maximum errors of approximately $1 \%$ by a linear least squares analysis for the ranges $1 \leqq \alpha \leqq 200,200 \leqq \alpha \leqq 10,000,10,000 \leqq \alpha \leqq 100,000$ with $E=14$ for a CDC 6600 computer. If $\alpha \leqq 1$, we increase $\alpha$ by one and proceed for $\alpha>1$ entering the backward recursive loop at least one time, even when $x<1+\alpha$. For the lower tail, the brackets \{ \} in the exponentials can be tested for underflow. As described, $\gamma / \Gamma$ is a millisecond significant digit routine on the CDC 6600 .

$z=\max \{0, \beta-1\}$ provides a starting value for Newton's iteration in the solution of (8) for $z_{0}$ when $\mu>1$. Then,

$$
f^{\prime}(z)=-\sum_{k=1}^{n} \frac{\alpha_{k} c_{k}}{\left(1+\alpha_{k}\right)} \frac{\Phi\left(2,2+\alpha_{k} ; c_{k} z\right)}{\Phi^{2}\left(1,1+\alpha_{k} ; c_{k} z\right)}
$$

is needed in addition to (8) and

$$
y^{\prime \prime}\left(z_{0}\right)=\left[f^{\prime}\left(z_{0}\right)-1\right] / z_{0} .
$$

The convergence criteria for Newton's method need not be severe since $z_{0}$ is not needed very accurately.

For the bound $B_{1}$, the procedure described above is used in computing the ratios $\gamma / \Gamma$ needed in (4). The bound $B_{2}$ can be computed by

$$
B_{2}=\frac{\Gamma(\beta, x)}{\Gamma(\beta)}=1-\frac{\gamma(\beta, x)}{\Gamma(\beta)}
$$

when $I_{n}$ is relatively large (say $\geqq 0.1$ as measured a priori by (5)) even though several 
significant figures will be lost when the relative error test is severe. However, $\boldsymbol{B}_{\mathbf{2}}$ must be generated another way when $I_{n}$ is much smaller because all significance will be lost in this relation and the relative error test cannot be met. In these cases, the continued fraction

$$
\begin{gathered}
S_{\nu+1}=0, \\
S_{k}=x+\frac{\left(k-\beta_{0}\right) S_{k+1}}{k+S_{k+1}}, \quad k=\nu, \nu-1, \cdots, 1, \\
A_{1}=e^{x} x^{-\beta_{0}} \frac{\Gamma\left(\beta_{0}, x\right)}{\Gamma\left(\beta_{0}\right)}=\frac{1 / \Gamma\left(\beta_{0}\right)}{S_{1}} \cdot S, \quad 0<\beta_{0} \leqq 1,
\end{gathered}
$$

with the forward recurrence scheme for $\beta=\beta_{0}+N>1$,

$$
\begin{aligned}
T_{1} & =\frac{1 / \Gamma\left(\beta_{0}\right)}{\beta_{0}} \cdot S \\
A_{k+1} & =A_{k}+T_{k}, \\
T_{k+1} & =T_{k} \cdot x /\left(\beta_{0}+k\right), \quad k=1,2, \cdots, N=\left\{\begin{array}{c}
{[\beta]} \\
{[\beta]-1}
\end{array}\right\} \\
\frac{\Gamma(\beta, x)}{\Gamma(\beta)} & =\left(\exp \left\{-x+\beta_{0} \ln x\right\} / S\right) \cdot A_{N+1}
\end{aligned}
$$

has proven satisfactory. $S$ is a positive scale factor on the order of the underflow limit of the machine.

The asymptotic form (5) produced the right order of magnitude for $I_{n}$ in all cases tested with one to three significant digits quite common.

In the case that $\alpha_{k}=\alpha$ and $c_{k}=c$ for all $k$, the computer time required is much less since the product in (1) reduces to a simple power and only one gamma ratio need be evaluated for each $z$. In this case, the sums (8), (10), and (11) as well as the product in (4) collapse to one term. In [13], Gupta obtained (1) for this case and gave percent point tables for $c$ with $\alpha=\beta=$ integer for $1 \leqq \alpha \leqq 25$ and $1 \leqq n \leqq 10$. Samples from these tables were checked using this procedure on a CDC 6600 computer with a Romberg integration routine. The functional relationships were extended in tabular form in [2] to values of $n$ up to 1000 for various. values of $c$ in $1.1 \leqq c \leqq 3.0$. Values of $\beta=\alpha$ up to 4700 appear in the body of the tables.

Special Cases. If one writes the gamma functions as integrals and exchanges the order of integration, the result is an $n$-fold integral

$$
I_{n}=c_{1}^{\alpha_{1}} \cdots c_{n}^{\alpha_{n}} \frac{\Gamma(\mu)}{\Gamma(\beta) \Gamma\left(\alpha_{1}\right) \cdots \Gamma\left(\alpha_{n}\right)} \int_{0}^{1} \cdots \int_{0}^{1} \frac{v_{1}^{\alpha_{1}-i} \cdots v_{n}^{\alpha_{n}-1} d v_{1} \cdots d v_{n}}{\left(1+c_{1} v_{1}+\cdots+c_{n} v_{n}\right)^{\mu}}
$$

which can be identified as an $n$-fold Lauricella sum $F_{A}$ of $n$ variables [4, pp. 114, 115]. Although special manipulative formulae exist for this function, the results do not appear numerically advantageous for $n>1$. However, this integral is also an inverted Dirichlet distribution and some recursive results are given in [27] for $\alpha_{k}$ even.

For $n=1$, we have very simple results in terms of the incomplete beta distribution 


$$
\begin{aligned}
& \int_{0}^{\infty} \frac{e^{-z} z^{\beta-1}}{\Gamma(\beta)} \frac{\gamma(\alpha, c z)}{\Gamma(\alpha)} d z=I_{x}(\alpha, \beta), \\
& \int_{0}^{\infty} \frac{e^{-s} z^{\beta-1}}{\Gamma(\beta)} \frac{\Gamma(\alpha, c z)}{\Gamma(\alpha)} d z=I_{1-x}(\beta, \alpha), \\
& x=c /(1+c), \quad 1-x=1 /(1+c) .
\end{aligned}
$$

For $n=2$, the Lauricella function reduces to the Appell $F_{2}$ function of two variables $[4$, p. 28], [8, p. 230]. This function also has special manipulative properties, but some additional formulae in terms of readily computable functions can be derived. We start with

$$
I_{2}(\hat{\alpha}, \hat{c}, \beta)=\int_{0}^{\infty} \frac{e^{-s} z^{\beta-1}}{\Gamma(\beta)} \frac{\gamma\left(\alpha_{1}, c_{1} z\right)}{\Gamma\left(\alpha_{1}\right)} \frac{\gamma\left(\alpha_{2}, c_{2} z\right)}{\Gamma\left(\alpha_{2}\right)} d z
$$

and use various representations of $\gamma\left(\alpha_{1}, c_{1} z\right)$ and $\gamma\left(\alpha_{2}, c_{2} z\right)$ to derive sums in terms of incomplete beta distributions. Note the symmetry in $\left(\alpha_{1}, c_{1}\right)$ and $\left(\alpha_{2}, c_{2}\right)$. The representations presented below are obtained by applying (7) in addition to

$$
\frac{\gamma(\alpha, x)}{\Gamma(\alpha)}=1-\frac{\Gamma(\alpha, x)}{\Gamma(\alpha)}, \quad \frac{\Gamma(n, x)}{\Gamma(n)}=e^{-x} \sum_{k=0}^{n-1} \frac{x^{k}}{k !},
$$

to one or both gamma functions followed by reduction with (12). The results are

(14) $I_{2}=I_{u}\left(\alpha_{1}, \beta\right)-\frac{(1-u)^{\beta}}{\alpha_{1}} \frac{\Gamma\left(\beta+\alpha_{1}\right)}{\Gamma(\beta) \Gamma\left(\alpha_{1}\right)} \sum_{k=0}^{\infty} \frac{\left(\beta+\alpha_{1}\right)_{k}}{\left(1+\alpha_{1}\right)_{k}} u^{k+\alpha_{1}} I_{1-y}\left(k+\beta+\alpha_{1}, \alpha_{2}\right)$,

$$
\begin{aligned}
I_{2} & =\frac{(1-u)^{\beta}}{\alpha_{1}} \frac{\Gamma\left(\beta+\alpha_{1}\right)}{\Gamma(\beta) \Gamma\left(\alpha_{1}\right)} \sum_{k=0}^{\infty} \frac{\left(\beta+\alpha_{1}\right)_{k}}{\left(1+\alpha_{1}\right)_{k}} u^{k+\alpha_{1}} I_{y}\left(\alpha_{2}, k+\beta+\alpha_{1}\right), \\
u & =c_{1} /\left(1+c_{1}\right), \quad 1-u=1 /\left(1+c_{1}\right), \\
y & =c_{2} /\left(1+c_{1}+c_{2}\right), \quad 1-y=\left(1+c_{1}\right) /\left(1+c_{1}+c_{2}\right),
\end{aligned}
$$

$$
\begin{aligned}
I_{2} & =I_{v}\left(\alpha_{2}, \beta\right)-(1-u)^{\beta} \sum_{k=0}^{\alpha_{1}-1} \frac{(\beta)_{k}}{k !} u^{k} I_{y}\left(\alpha_{2}, k+\beta\right), \\
v & =c_{2} /\left(1+c_{2}\right), \quad 1-v=1 /\left(1+c_{2}\right), \quad \alpha_{1} \text { a positive integer, } \\
I_{2} & =1-I_{1-u}\left(\beta, \alpha_{1}\right)-I_{1-,}\left(\beta, \alpha_{2}\right)
\end{aligned}
$$$$
+(1-u)^{\beta} \sum_{k=0}^{\alpha_{1}-1} \frac{(\beta)_{k}}{k !} u^{k} I_{1-y}\left(k+\beta, \alpha_{2}\right), \quad \alpha_{1} \text { a positive integer. }
$$

(15) was obtained by Tiao and Guttman in [27]. Sequences of incomplete beta functions $I_{x}(p+k, q)$ or $I_{x}(p, q+k), k=0,1,2, \cdots$, can easily be generated by the methods described in [1], [3], [11], and [12]. The series in (13) and (14) will be slowly convergent if $\beta$ is large and $c_{1}$ is large. On the other hand, (15) and (16) are computationally appealing if either $\alpha_{1}$ or $\alpha_{2}$ is an integer due to the symmetry in $\left(\alpha_{1}, c_{1}\right)$ and $\left(\alpha_{2}, c_{2}\right)$. The series (13) has all positive terms and one expects significant digit results. The incomplete beta function in (14) helps convergence since $I_{x}(p+k, q)$ decreases monotonically to zero for $k \rightarrow \infty$ while $I_{x}(p, q+k)$ increases monotonically to 1 for $k \rightarrow \infty$. 
If $P_{a b}\left(a_{1} \leqq F_{1} \leqq b_{1}, \cdots, a_{n} \leqq F_{n} \leqq b_{n} \mid \beta, \alpha_{k}, k=1, \cdots, n\right)$ is desired, expansion of the product in

$$
P_{a b}=\int_{0}^{\infty} \frac{e^{-z^{\beta} z^{\beta-1}}}{\Gamma(\beta)} \prod_{k=1}^{n} \frac{\gamma\left(\alpha_{k}, c_{k b} z\right)-\gamma\left(\alpha_{k}, c_{k a} z\right)}{\Gamma\left(\alpha_{k}\right)} d z,
$$

with

$$
c_{k b}=\alpha_{k} b_{k} / \beta, \quad c_{k a}=\alpha_{k} a_{k} / \beta,
$$

gives results in terms of $I_{n}$. The special case, where $\alpha_{k}=\alpha, a_{k}=a, b_{k}=b$, is

$$
P_{a b}=\sum_{k=0}^{n}(-1)^{k}\left(\begin{array}{l}
n \\
k
\end{array}\right) I_{n}\left(\hat{\alpha}, \hat{c}_{k}, \beta\right),
$$

where

$$
I_{n}\left(\alpha, \hat{c}_{k}, \beta\right)=\int_{0}^{\infty} \frac{e^{-z} z^{\beta-1}}{\Gamma(\beta)}\left[\frac{\gamma\left(\alpha, c_{b} z\right)}{\Gamma(\alpha)}\right]^{n-k}\left[\frac{\gamma\left(\alpha, c_{a} z\right)}{\Gamma(\alpha)}\right]^{k} d z
$$

and $c_{b}=\alpha b / \beta, c_{a}=\alpha a / \beta$. In this form, only two gamma ratios need to be evaluated for each value of $z$ in (11). On the CDC 6600, with the relative error requirements on the order of $5 \times 10^{-6}$ and $n=20$, one can expect each integral of (17) to take on the order of 0.5 to 5 seconds with Romberg integration, the longer times occurring for larger $k$ values. It is possible that an adaptive Romberg routine could reduce the computer time.

The Correlated Bivariate $F$ Distribution. The bivariate $\chi^{2}$ distribution with correlation $\rho^{2}$ for random variables $X_{1}, X_{2}$, each with $m$ degrees of freedom, can be represented as [22]

$g\left(x_{1}, x_{2}\right)=\left(1-\rho^{2}\right)^{m / 2} \sum_{i=0}^{\infty} \frac{(m / 2)_{i}}{i !} \rho^{2 i} \frac{\left(x_{1} x_{2}\right)^{m / 2+i-1} \exp \left\{-\left(x_{1}+x_{2}\right) /\left[2\left(1-\rho^{2}\right)\right]\right\}}{\left[2^{m / 2+i}\left(1-\rho^{2}\right)^{m / 2+i} \Gamma(m / 2+i)\right]^{2}}$.

If $Y$ is $\chi^{2}$ with $n$ degrees of freedom

$$
f(y)=\frac{1}{2^{n / 2} \Gamma(n / 2)} y^{n / 2-1} e^{-y / 2}
$$

and $Y$ is independent of $X_{1}$ and $X_{2}$, then the variables

$$
F_{i}=\frac{X_{i} / m}{Y / n}, \quad i=1,2,
$$

have a joint cumulative distribution given by

$$
\begin{aligned}
P\left(0 \leqq F_{1} \leqq f_{1}, 0\right. & \left.\leqq F_{2} \leqq f_{2} \mid \rho, m, n\right) \\
& =\int_{0}^{\infty} f(y) \int_{0}^{f_{2} m y / n} \int_{0}^{f_{1} m y / n} g\left(x_{1}, x_{2}\right) d x_{1} d x_{2} d y .
\end{aligned}
$$

In series form,

$$
P=\left(1-\rho^{2}\right)^{m / 2} \sum_{i=0}^{\infty} \frac{(m / 2)_{i}}{i !} \rho^{2 i} I_{2}\left(\alpha_{i}, \hat{c}, \beta\right),
$$


where $I_{2}\left(\hat{\alpha}_{i}, \hat{c}, \beta\right)$ is given by (3), (13), (14), (15) or (16), and

$$
\beta=n / 2, \quad \hat{c}=\left(\frac{f_{1} m}{n\left(1-\rho^{2}\right)}, \frac{f_{2} m}{n\left(1-\rho^{2}\right)}\right), \quad \hat{\alpha}_{i}=\left(\frac{m}{2}+i, \frac{m}{2}+i\right) .
$$

There is symmetry in the pairs $\left(\alpha_{1}, c_{1}\right),\left(\alpha_{2}, c_{2}\right)$. For use in (1), $\alpha_{1}=\alpha_{2}=m / 2+i$ and there is complete symmetry in $c_{1}$ and $c_{2}$. Equations (13) and (14), together with the quadrature in (3), do not require either $\alpha_{1}$ or $\alpha_{2}$ to be integers. One always expects significant digits from the positive series (13) and the quadrature of (3). In these cases, (19) also yields significant digits.

When $m$ is even the formula

$$
\left(1-\rho^{2}\right)^{-m / 2}=\sum_{i=0}^{\infty} \frac{(m / 2)_{i}}{i !} \rho^{2 i}, \quad 0 \leqq \rho^{2}<1,
$$

with (19) and (16) gives

$$
P=1-\left(1-\rho^{2}\right)^{m / 2} \sum_{i=0}^{\infty} \frac{(m / 2)_{i}}{i !} \rho^{2 i} J_{2}\left(\hat{\alpha}_{i}, \hat{c}, \beta\right),
$$

where $\beta=n / 2, \alpha_{1}=\alpha_{2}=m / 2+i$ in

$$
\begin{aligned}
J_{2}(\hat{\alpha}, \hat{c}, \beta)= & I_{1-u}\left(\beta, \alpha_{1}\right)+I_{1-。}\left(\beta, \alpha_{2}\right) \\
& -(1-u)^{\beta} \sum_{k=0}^{\alpha_{1}-1} \frac{(\beta)_{k}}{k !} u^{k} I_{1-\nu}\left(\beta+k, \alpha_{2}\right), \quad \alpha_{1} \text { a positive integer. }
\end{aligned}
$$

At first glance, one might expect faster convergence for $\rho$ close to 1 . However the experiments detailed below indicate otherwise. Other series representations can be obtained by exchanging the order of summation in (19) and (20).

Computations with (19) and (20) were done for the first quadrant of the $f_{1}, f_{2}$ plane for $m, n=2,10,50$ and $\rho=0.1,0.5,0.9$. No extensive testing of (19) with (13) or (14) was done because of the slow convergence when $\beta$ and $c_{1}$ are large. Although (19) with the quadrature in (3) worked for small $\rho$, the speed of (19) (with (15)) and (20) ruled out the quadrature as a satisfactory procedure for $m$ even. The series (19) and (20) were truncated when a term was less than $5 \times 10^{-6}$ of the accumulated sum (always less than 225 terms). The agreement was generally four significant figures with slight discrepancies in the fifth digit for some $\rho=0.9$ cases. When $P<0.1$ in (20), only absolute accuracy was obtained.

The comparisons were made on a CDC 6600 computer with incomplete beta sequences generated according to the method described in [1] and [2]. 675 comparisons inside the radii listed in Table 1 showed (19) to be approximately three times faster than (20) with running times of 2 and 6 minutes respectively. The corresponding time for the quadrature in (3) for the 450 cases associated with $\rho=0.1$ and $\rho=0.5$ was on the order of 30 minutes with comparable relative error requirements. The accuracy with which $z_{0}$ was isolated in (8) seemed to have a strong influence on the overall speed of computation in (3).

Acknowledgement. The authors wish to acknowledge the assistance of Mrs. Sharon L. Daniel in coding the numerical experiments for the CDC 6600 computer. 


\section{TABLE 1}

Radii in the $f_{1}, f_{2}$ Plane where $P=0.95$ on the $45^{\circ}$ Rav for $\rho=0.1,0.5,0.9$

\begin{tabular}{|c|c|c|c|}
\hline$m \backslash n$ & 2 & 10 & 50 \\
\hline \multirow[t]{3}{*}{2} & 40.70 & 7.52 & 5.58 \\
\hline & 38.90 & 7.34 & 5.49 \\
\hline & 32.91 & 6.64 & 5.07 \\
\hline \multirow[t]{3}{*}{10} & 34.22 & 4.97 & 3.25 \\
\hline & 33.33 & 4.88 & 3.2 \\
\hline & 30.39 & 4.58 & 3.07 \\
\hline \multirow[t]{3}{*}{50} & 30.63 & 4.09 & 2.44 \\
\hline & 30.23 & 4.05 & 2.42 \\
\hline & 28.89 & 3.90 & 2.35 \\
\hline
\end{tabular}

Applied Mathematics Division

Sandia Laboratories

Albuquerque, New Mexico 87115

Mathematics Department

University of Kansas

Lawrence, Kansas 66044

1. D. E. Amos, Significant Digit Computation of Certain Distribution Functions, Proc. Sympos. on Empirical Bayes Estimation and Computing in Statistics (Texas Tech. Univ., Lubbock, Texas, 1969), Texas Tech. Press, Lubbock, Tex., 1970, pp. 165-180.

2. D. E. Amos \& S. L. DANIEL, Evaluation of Probabilities Associated with a Fixed Sample Selection Procedure, Sandia Corporation Research Report SC-RR-69-752 (available through Division 3151, Sandia Corporation, Albuquerque, N. M. 87115).

3. D. E. Amos \& S. L. Daniel, Significant Digit Incomplete Beta Ratios, Sandia Corporation Development Report SC-DR-69-591 (available through Division 3151, Sandia Corporation, Albuquerque, N. M. 87115).

4. P. APPELl \& J. KAMPÉ DE FÉRIET, Fonctions Hypergéométriques et Hypersphériques Polynomes d'Hermite, Gauthier-Villars, Paris, 1926.

5. L. D. BRoEmeling, "Confidence regions for variance ratios of random models," J. Amer. Statist. Assoc., v. 64, 1969, pp. 660-664. 330.

6. V. CHEW, "Simultaneous prediction intervals," Technometrics, v. 10, 1968, pp. 323-

7. B. Epstern, "Estimation from life test data," Technometrics, v. 2, 1960, pp. 447-454.

8. A. ERDÉLYI, ET AL., Higher Transcendental Functions. Vol. 1, McGraw-Hill, New York, 1953. MR 15, 419.

9. A. ERDÉLYI, ET AL., Higher Transcendental Functions. Vol. 2, McGraw-Hill, New York, 1953. MR 15, 419.

10. J. L. Folks \& C. H. ANTLE, "Straight line confidence regions for linear models," J. Amer. Statist. Assoc., v. 62, 1967, pp. 1365-1374. MR 36 \#4722.

11. W. GAUTSCHI, "Algorithm 222-incomplete beta function ratios," Comm. Assoc. Comput. Mach., v. 7, 1964, pp. 143-144; "Certification of Algorithm 22," ibid., 1964, p. 244.

12. W. GAUTSCHI, "Computational aspects of three-term recurrence relations," SIAM Rev., v. 9, 1967, pp. 24-82. MR 35 \#3927.

13. S. S. GUPTA, "On a selection and ranking procedure for gamma populations," Ann. Inst. Statist. Math., v. 14, 1962/63, pp. 199-216. MR 28 \#690.

14. S. S. Gupta \& M. SobEL, "On the smallest of several correlated $F$ statistics," Biometrika, v. 49, 1962, pp. 509-523. MR 28 \#3514.

15. JOHN E. HEWETT, "A Note on prediction intervals based on partial observations in certain life test experiments," Technometrics, v. 10, 1968, pp. 850-853.

16. J. E. HeWETt \& W. G. Bulgren, "Inequalities for some multivariate $f$-distributions with applications," Technometrics, v. 13, 1971, pp. 397-402.

17. D. R. JENSEN, "An inequality for a class of bivariate chi-square distributions," $J$. Amer. Statist. Assoc., v. 64, 1969, pp. 333-336. MR 39 \#2241. 
18. C. G. KHATRI, "On certain inequalities for normal distributions and their applications to simultaneous confidence bounds," Ann. Math. Statist., v. 38, 1967, pp. 1853-1867. MR 36 \#3452.

19. A. W. Kimball, "On dependent tests of significance in the analysis of variance," Ann. Math. Statist., v. 22, 1951, pp. 600-602. MR 13, 368.

20. P. R. KRISHNAiaH, "On the simultaneous ANOVA and MANOVA tests," Ann. Inst. Statist. Math., v. 17, 1965, pp. 35-53. MR 33 \#17.

21. P. R. KRISHNAIAH \& J. V. ARMITAge, Probability Integrals of the Multivariate F Distribution, with Tables and Applications, ARL 65-236 Aerospace Research Laboratories, Wright-Patterson Air Force Base, Ohio, November 1965.

22. P. R. Krishnaiah, P. Hagis \& L. Steinberg, "A note on the bivariate chi distribution,"SIAM Rev., v. 5, 1963, pp. 140-144. MR 27 \#3038.

23. J. NADLER, "Inverse binomial sampling plans when an exponential distribution is sampled with censoring," Ann. Math. Statist., v. 31, 1960, pp. 1201-1204. MR 22 \#11478.

24. K. R. NaIR, "The studentized form of the extreme mean square test in the analysis of variance," Biometrika, v. 35, 1948, pp. 16-31. MR 9,601.

25. D. B. OWEN, Some Current Problems In Statistics Requiring Numerical Results, Proc. Sympos. on Empirical Bayes Estimation and Computing Statistics (Texas Tech. Univ., Lubbock, Texas, 1969), Texas Tech. Press, Lubbock, Tex., 1970, pp. 155-164.

26. PAUl Terrel POPE, On the Stepwise Construction of a Prediction Equation, Technical Report No. 37, Dept. of Statistics, SMU, 1969.

27. G. C. TIAO \& I. Gutrman, "The inverted Dirichlet distribution with applications," J. Amer. Statist. Assoc., v. 60, 1965, pp. 793-805. MR 32 \#3177. 